\title{
Three-nucleon force contribution in the distorted-wave theory of $(d, p)$ reactions
}

\author{
N. K. Timofeyuk \\ Department of Physics, Faculty of Engineering and Physical Sciences, University of Surrey, Guildford, GU2 7XH, United Kingdom
}

(Received 1 December 2017; published 2 May 2018)

\begin{abstract}
The distorted-wave theory of $A(d, p) B$ reactions, widely used to analyze experimental data, is based on a Hamiltonian that includes only two-nucleon interactions. However, numerous studies of few-nucleon systems and many modern developments in nuclear structure theory show the importance of the three-nucleon $(3 N)$ force. The purpose of this paper is to study the contribution of the $3 N$ force of the simplest possible form to the $A(d, p) B$ reaction amplitude. This contribution is given by a new term that accounts for the interaction of the neutron and proton in the incoming deuteron with one of the target nucleons. This term involves a new type of nuclear matrix elements containing an infinite number of target excitations in addition to the main part associated with the traditional overlap function between $A$ and $B$. The nuclear matrix elements are calculated for double-closed shell targets within a mean field theory where target excitations are shown to be equivalent to exchanges between valence and core nucleons. These matrix elements can be readily incorporated into available reaction codes if the $3 N$ interaction has a spin-independent zero-range form. Distorted-wave calculations are presented for a contact $3 N$ force with the volume integral fixed by the chiral effective field theory at the next-to-next-to-leading order. For this particular choice, the $3 N$ contribution is noticeable, especially at high deuteron incident energies. No $3 N$ effects are seen for incident energies below the Coulomb barrier. The finite range can significantly affect the $3 N$ contribution to the $(d, p)$ cross sections. Finite-range studies require new formal developments and, therefore, their contribution is preliminarily assessed within the plane-wave Born approximation, together with sensitivity to the choice of the deuteron model.
\end{abstract}

DOI: 10.1103/PhysRevC.97.054601

\section{INTRODUCTION}

Modern experiments with radioactive beams use nucleon transfers, for example, deuteron stripping reactions $A(d, p) B$, to pin down the spectroscopy of various isotopes from different areas of the nuclear chart. To obtain information such as spectroscopic factors and asymptotic normalization coefficients, they have to rely on a comparison between experimental and theoretical cross sections calculated using direct transfer reaction theory. Systematical uncertainties of the calculated cross sections have been reviewed in [1]. More recently, new theoretical uncertainties associated with $n-p$ interaction used in adiabatic treatment of deuteron breakup have been identified [2,3]. However, one potential source of uncertainties - the contribution from the three-nucleon $(3 N)$ force-has never been considered before. Given that many modern developments in nuclear structure theory show the importance of the $3 N$ force, estimates of the possible $3 N$ contribution in $(d, p)$ reactions are timely.

The theory of direct transfer reactions was developed in the 1960s and 1970s. The starting point of this theory is a many-body Hamiltonian with two-body interactions [4,5]. The most popular approximation of such a theory-the distorted-wave Born approximation (DWBA) - is designed to express quantities entering the reaction amplitude via those calculated using information obtained phenomenologically from somewhere else. Thus, the DWBA description of an $A(d, p) B$ reaction relies on knowledge of optical potentials fitted to describe $d+A$ and $p+B$ elastic scattering data. Such phenomenological potentials should already include the contributions from the $3 N$ force since experimental cross sections include its effects. Going beyond DWBA, one has to account for deuteron breakup, which is usually done within the adiabatic distorted-wave approximation (ADWA) by solving the $A+n+p$ Schrödinger equation with $p$ - $A$ and $n$ - $A$ phenomenological optical potentials [6]. Such potentials already include the $3 N$ contribution. The $3 N$ force that involves $n, p$, and one of the nucleons from $A$ should give a new contribution to the $d$ - $A$ adiabatic potential. However, this force will have to compete with an effective three-body $p-n-A$ force, which arises when the neutron (proton) in the incoming deuteron excites the target $A$ and these excitations are passed on to the proton (neutron) and then back to neutron (proton) in all orders [7]. Therefore, the contribution to the $d$ - $A$ distorting potential from a bare $3 N$ force should not be considered independently of the $p-n-A$ effective three-body force induced by two-nucleon $(2 N)$ interactions. Such considerations are a challenging task.

The aim of the present paper is to point out that the $3 \mathrm{~N}$ force should create an additional contribution to the $(d, p)$ amplitude within both the DWBA and the ADWA frameworks in the vertex of these amplitudes and to calculate this contribution using the simplest possible form of the $3 N$ force. With the twobody force only, the distorted-wave amplitudes are written as

$$
T_{(d, p)}=\left\langle\Psi_{M_{B}}^{J_{B}} \psi_{M_{p}}^{J_{p}} \chi_{\boldsymbol{k}_{p}}\left(\boldsymbol{R}_{p}\right)\left|V_{n p}(\boldsymbol{r})\right| \Psi_{M_{A}}^{J_{A}} \psi_{M_{d}}^{J_{d}} \chi_{\boldsymbol{k}_{d}}\left(\boldsymbol{R}_{d}\right)\right\rangle
$$




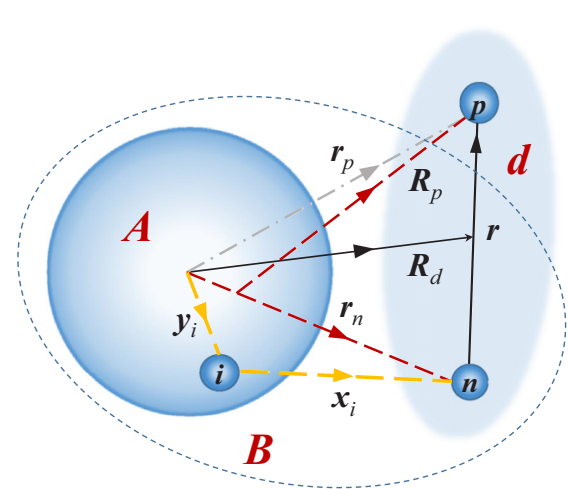

FIG. 1. Definition of coordinates for an $A(d, p) B$ reaction.

where $\Psi_{M_{A}}^{J_{A}}\left(\Psi_{M_{B}}^{J_{B}}\right)$ is the wave function of the target (daughter) nucleus with the angular momentum $J_{A}\left(J_{B}\right)$ and its projection $M_{J_{A}}\left(M_{J_{B}}\right), \psi_{M_{d}}^{J_{d}}$ is the incoming deuteron wave function, $\psi_{M_{p}}^{J_{p}}$ is the outgoing proton spin function and $J_{d}\left(J_{p}\right)$ and $M_{J_{d}}\left(M_{J_{p}}\right)$ are the spins and spin projections of the deuteron (proton). Also, $\chi_{\boldsymbol{k}_{d}}\left(\boldsymbol{R}_{d}\right)$ is the deuteron distorted wave with momentum $\boldsymbol{k}_{d}$, obtained either in DWBA or in ADWA, and $\chi_{\boldsymbol{k}_{p}}\left(\boldsymbol{R}_{p}\right)$ is the proton distorted wave in the exit channel with momentum $\boldsymbol{k}_{p}$, while $V_{n p}$ is the interaction between $n$ and $p$ in the deuteron. The definitions of all the coordinates are given in Fig. 1. The paper will discuss an additional term which arises in $T_{(d, p)}$ when a $3 N$ force is included. This term has the same structure as Eq. (1) but with $V_{n p}$ replaced by $\sum_{i \in A} W_{i n p}$, where $W_{i n p}$ is the interaction between $n$ and $p$ from the deuteron and the nucleon $i$ from the target.

The present paper calculates the contributions from $\sum_{i \in A} W_{i n p}$ using a simple hypercentral model for the $3 N$ force. It starts with discussion in Sec. II of the distorted-wave $(d, p)$ transfer amplitude, that contains this term. Then Sec. III considers the plane-wave approximation of this amplitude. It points out that a new type of nuclear matrix elements (form factors) is needed for $(d, p)$ calculations. These form factors are considered in Sec. IV within a mean field approach. Sections V and VI describe plane-wave and distorted-wave calculations with the zero-range $3 N$ force, respectively. Section VII studies finite-range effects from the $3 N$ forces within the plane-wave Born approximation and Sec. VIII summarizes the results obtained and draws conclusions.

\section{DISTORTED-WAVE AMPLITUDE WITH $3 N$ VERTEX}

The starting point of $A(d, p) B$ direct reaction theory is the Schrödinger equation for an $A+2$-body wave function $\Phi_{d}^{(+)}$ satisfying the asymptotic condition of deuteron plane-wave motion relative to the target $A$. The $(d, p)$ amplitude $T_{d, p}$ is found from the asymptotics of the projection of $\Phi_{d}^{(+)}$into the wave function of nucleus $B$. Following the reasoning of $[4,5]$ one can show that in the case when the $A+2$ body Hamiltonian contains $3 N$ interactions, $\sum_{i<j<k} W_{i j k}$, the amplitude $T_{d, p}$ in the Born approximation is written as

$$
\begin{aligned}
T_{(d, p)}= & \left\langle\Psi_{M_{B}}^{J_{B}} \psi_{M_{p}}^{J_{p}} \chi_{\boldsymbol{k}_{p}}\left(\boldsymbol{R}_{p}\right)\right| \sum_{i \in B} V_{p i}+\sum_{i<j \in B} W_{p i j} \\
& -U_{p}\left(\boldsymbol{R}_{p}\right)\left|\Psi_{M_{A}}^{J_{A}} \psi_{M_{d}}^{J_{d}} \chi_{\boldsymbol{k}_{d}}\left(\boldsymbol{R}_{d}\right)\right\rangle,
\end{aligned}
$$

where the auxiliary potential $U_{p}$ is used to generate the distorted-wave $\chi_{k_{p}}$. It is always assumed that $U_{p}$ is the $p+B$ optical potential that reproduces elastic scattering in the exit proton channel. However, it has been pointed out in $[8,9]$ that $U_{p}$ is rather an optical potential for the $p+A$ scattering. For sufficiently heavy nuclei, using the $p+B$ optical potential instead of the $p+A$ one does not make any significant difference. Therefore, following usual practice, one can assume that

$$
\sum_{i \in A} V_{p i}+\sum_{i<j \in A} W_{p i j}-U_{p}\left(\boldsymbol{R}_{p}\right) \approx 0 .
$$

This assumption should work better than the standard assumption $\sum_{i \in A} V_{p i}-U_{p}\left(\boldsymbol{R}_{p}\right) \approx 0$, made in $(d, p)$ reaction theory because phenomenological optical potentials are fitted to describe experimental elastic scattering data in which $3 N$ effects are always present. With (3) and (2) one gets

$$
T_{(d, p)}=T_{(d, p)}^{2 N}+T_{(d, p)}^{3 N},
$$

where $T_{(d, p)}^{2 N}$ is given by Eq. (1), and

$$
T_{(d, p)}^{3 N}=\left\langle\Psi_{M_{B}}^{J_{B}} \psi_{M_{p}}^{J_{p}} \chi_{\boldsymbol{k}_{p}}\left(\boldsymbol{R}_{p}\right)\left|\sum_{i \in A} W_{i p n}\right| \Psi_{M_{A}}^{J_{A}} \psi_{M_{d}}^{J_{d}} \chi_{\boldsymbol{k}_{d}}\left(\boldsymbol{R}_{d}\right)\right\rangle .
$$

There is an important difference between $T_{(d, p)}^{2 N}$ and $T_{(d, p)}^{3 N}$. In the former case, $V_{n p}$ does not depend on the internal coordinates of $A$ and, therefore, $T_{d, p}^{(2 N)}$ contains the overlap function $\left\langle\Psi_{M_{B}}^{J_{B}} \mid \Psi_{M_{A}}^{J_{A}}\right\rangle$, usually parametrized via a single-particle neutron wave function times the square root of the spectroscopic factor. This parametrization is the cornerstone for extracting spectroscopic factors from experimental $(d, p)$ cross sections. In contrast, the $3 N$ force affects internal coordinates of $A$ in $T_{(d, p)}^{3 N}$, and the form factor $\left\langle\Psi_{M_{B}}^{J_{B}}\left|\sum_{i \in A} W_{i p n}\right| \Psi_{M_{A}}^{J_{A}}\right\rangle$ cannot be parametrized in term of a single spectroscopic factor corresponding to the overlap $\left\langle\Psi_{M_{B}}^{J_{B}} \mid \Psi_{M_{A}}^{J_{A}}\right\rangle$. This could introduce new theoretical uncertainties into spectroscopic factor extraction if the contribution from the $3 N$ force is important. These effects are estimated below using several approximations, the starting point of which is the plane-wave Born approximation (PWBA).

\section{PLANE-WAVE APPROXIMATION FOR $(d, p)$ AMPLITUDE WITH $3 N$ VERTEX}

Although the PWBA does not give the correct magnitude of $(d, p)$ cross sections, it catches correctly the general feature that their angular distributions are related to the Fourier transform of the single-particle wave function of the neutron which has been transferred from deuteron to the target $A$. Besides, any distorted wave can be expanded over plane waves [10] and, indeed, one of the DWBA codes available to the nuclear physics community, DWUCK5, is based on these plane-wave expansions [11]. Therefore, PWBA is a natural first step to 
assess the importance of the $3 N$ vertex in $(d, p)$ cross sections. The assessment is done here for a simple spin-independent $3 N$ force.

After replacing the proton and deuteron distorted wave by plane waves, the $(d, p)$ transition amplitude reads

$$
T_{(d, p)}^{\mathrm{PWBA}}=\left\langle\Psi_{M_{B}}^{J_{B}} \psi_{M_{p}}^{J_{p}} e^{i \boldsymbol{k}_{p} \boldsymbol{R}_{p}}|\mathcal{V}| \Psi_{M_{A}}^{J_{A}} \psi_{M_{d}}^{J_{d}} e^{i \boldsymbol{k}_{d} \boldsymbol{R}_{d}}\right\rangle
$$

where $\mathcal{V}=V_{n p}+\sum_{i \in A} W_{i p n}$. Using relations $\boldsymbol{R}_{d}=\boldsymbol{r}_{n}+\frac{1}{2} \boldsymbol{r}$ and $\boldsymbol{R}_{p}=\frac{A}{A+1} \boldsymbol{r}_{n}+\boldsymbol{r}$ (see Fig. 1) and introducing momentum vectors $\boldsymbol{k}=\frac{\boldsymbol{k}_{d}}{2}-\boldsymbol{k}_{p}$ and $\boldsymbol{q}=\boldsymbol{k}_{d}-\frac{A}{A+1} \boldsymbol{k}_{p}$, one gets for the $2 N$ force contribution the well-known expression

$$
T_{(d, p)}^{\mathrm{PWBA}, 2 N}=G_{M_{B} M_{A}}^{J_{B} J_{A}}(\boldsymbol{q}) D_{M_{p} M_{d}}^{J_{p} J_{d}}(\boldsymbol{k}),
$$

in which

$$
\begin{aligned}
D_{M_{p} M_{d}}^{J_{p} J_{d}}(\boldsymbol{k}) & =\int d \boldsymbol{r} e^{i \boldsymbol{k} \boldsymbol{r}}\left\langle\psi_{M_{p}}^{J_{p}}\left|V_{n p}\right| \psi_{M_{d}}^{J_{d}}\right\rangle, \\
G_{M_{B} M_{A}}^{J_{B} J_{A}}(\boldsymbol{q}) & =\int d \boldsymbol{r}_{n} e^{i \boldsymbol{q} \boldsymbol{r}_{n}}\left\langle\Psi_{M_{B}}^{J_{B}} \mid \Psi_{M_{A}}^{J_{A}}\right\rangle .
\end{aligned}
$$

The corresponding differential cross section is

$$
\frac{d \sigma^{\text {PWBA }, 2 N}}{d \Omega}=\frac{\mu_{p} \mu_{d}}{4 \pi^{2} \hbar^{4}} \frac{k_{p}}{k_{d}} \frac{\hat{J}_{B}^{2}}{2 \hat{J}_{A}^{2}} \frac{B}{4 \pi} D^{2}(k) \sum_{l j} G_{l j}^{2}(q),
$$

where $\mu_{d}$ and $\mu_{p}$ are reduced masses in the entrance deuteron and exit proton channels, respectively, $G_{l j}(q)$ is the radial part of the Fourier transform of the overlap function $\left\langle\Psi_{M_{B}}^{J_{B}} \mid \Psi_{M_{A}}^{J_{A}}\right\rangle$ corresponding to neutron orbital momentum $l$ and angular momentum $j, D(k)$ is the vertex formfactor, assumed here to have $s$-wave contribution only, and

$$
\begin{aligned}
& k=\left(\frac{1}{4} k_{d}^{2}-k_{p} k_{d} \cos \theta+k_{p}^{2}\right)^{1 / 2} \\
& q=\left[k_{d}^{2}-\frac{2 A}{A+1} k_{p} k_{d} \cos \theta+\left(\frac{A}{A+1}\right)^{2} k_{p}^{2}\right]^{1 / 2},
\end{aligned}
$$

in which $\theta$ is the scattering angle in the center of mass. The factor $B$ arises due to antisymmetrization [4]. Often it is included in the definition of the overlap function.

The $T_{(d, p)}^{\mathrm{PWBA}, 3 N}$ amplitude can be represented in a form similar to (7) if the $3 N$ force does not contain spin and isospin operators. This could be done by introducing the function

$$
\mathcal{D}_{M_{p} M_{d}}^{J_{p} J_{d}}\left(\boldsymbol{k}, \boldsymbol{x}_{i}\right)=\int d \boldsymbol{r} e^{i \boldsymbol{k} \boldsymbol{r}}\left\langle\phi_{M_{p}}^{J_{p}}\left|W_{i p n}\right| \psi_{M_{d}}^{J_{d}}\right\rangle,
$$

which is the folding of the $3 N$ force with the deuteron wave function. Being an analog of the deuteron vertex function $D_{M_{p} M_{d}}^{J_{p} J_{d}}(\boldsymbol{k})$ from Eq. (7), it also depends on the position of nucleon $i$ in the target $A$. The $3 N$ PWBA amplitude is then an average over momentum $\boldsymbol{p}$ of nucleon $i$ from $A$,

$$
T_{(d, p)}^{\mathrm{PWBA}, 3 N}=\frac{1}{(2 \pi)^{3}} \int d \boldsymbol{p} \mathcal{S}_{M_{A} M_{B}}^{J_{A} J_{B}}(\boldsymbol{q}, \boldsymbol{p}) \mathcal{D}_{M_{p} M_{d}}^{J_{p} J_{d}}(\boldsymbol{k}, \boldsymbol{p}),
$$

of the product of two structural quantities, one of which,

$$
\mathcal{D}_{M_{p} M_{d}}^{J_{p} J_{d}}(\boldsymbol{k}, \boldsymbol{p})=\int d \boldsymbol{x}_{i} e^{i \boldsymbol{p} \boldsymbol{x}_{i}} \mathcal{D}_{M_{p} M_{d}}^{J_{p} J_{d}}\left(\boldsymbol{k}, \boldsymbol{x}_{i}\right),
$$

is associated with deuteron and $3 N$ forces, acting as an effective two-body force, and another one,

$$
\mathcal{S}_{M_{A} M_{B}}^{J_{A} J_{B}}(\boldsymbol{q}, \boldsymbol{p})=\int d \boldsymbol{r}_{n} e^{i \boldsymbol{q} \boldsymbol{r}_{n}}\left\langle\Psi_{M_{B}}^{J_{B}}\left|\sum_{i \in A} e^{-i \boldsymbol{p} \boldsymbol{x}_{i}}\right| \Psi_{M_{A}}^{J_{A}}\right\rangle
$$

is associated with the structure of $A$ and $B$.

Compared to the $2 N$ case, where the nuclear structure comes via $G_{M_{B} M_{A}}^{J_{B} J_{A}}(\boldsymbol{q})$, the structural quantity $\mathcal{S}_{M_{A} M_{B}}^{J_{A} J_{B}}(\boldsymbol{q}, \boldsymbol{p})$ is a more complicated object. By introducing expansion of the wave function of daughter nucleus $B$ onto a complete set of states of $A$, it can be written as

$$
\begin{aligned}
\mathcal{S}_{M_{A} M_{B}}^{J_{A} J_{B}}(\boldsymbol{q}, \boldsymbol{p})= & \sum_{\alpha J_{A}^{\prime} M_{A}^{\prime} l j m j}\left(j m_{j} J_{A}^{\prime} M_{A}^{\prime} \mid J_{B} M_{B}\right) \\
& \times I_{l j m_{j}}^{(\alpha) *}(\boldsymbol{p}-\boldsymbol{q}) F_{0 \alpha}^{J_{A}^{\prime} M_{A}^{\prime} J_{A} M_{A}}(\boldsymbol{p}),
\end{aligned}
$$

where $\alpha$ denotes an excited state of the target $A$ and $I_{l j m_{j}}^{(\alpha)}$ is the overlap function between this state and the state of interest in $B$ populated by $(d, p)$. Another quantity in Eq. (16),

$$
F_{0 \alpha}^{J_{A}^{\prime} M_{A}^{\prime} J_{A} M_{A}}(\boldsymbol{p})=\left\langle\Psi_{M_{A}^{\prime}}^{J_{A}^{\prime}}\left|\sum_{i \in A} e^{i \boldsymbol{p} \boldsymbol{y}_{i}}\right| \Psi_{M_{A}}^{J_{A}}\right\rangle
$$

is the transition form factor between the ground state of the target $A$ and an excited state $\alpha$, defined in terms of position $\boldsymbol{y}_{i}$ of the $i$ th nucleon with respect to the center of mass of $A$. The first term of expansion (16) contains the same overlap function that features in $T_{d, p}^{\mathrm{PWBA}, 2 N}$ and which is associated with the spectroscopic factor of $\langle B \mid A\rangle$. All other terms have magnitudes determined by spectroscopic amplitudes of overlaps $\left\langle B \mid A^{*}\right\rangle$ between $B$ and the excited states of $A$, thus invalidating the wide-spread assumption that the total $(d, p)$ cross section is proportional to the spectroscopic factor of $\langle B \mid A\rangle$.

The quantity $\mathcal{S}_{M_{A} M_{B}}^{J_{A} J_{B}}(\boldsymbol{q}, \boldsymbol{p})$ is new to nuclear physics. Its calculation requires knowledge of the many-body wave functions of $A$ and $B$, ideally obtained with the $3 N$ force for consistency, which is a challenging task. To have a first idea of this quantity, it is studied in the following section in a mean field approach.

\section{NUCLEAR TRANSITION FORM FACTORS IN THE MEAN FIELD APPROACH}

Here and below it is assumed that the target $A$ is a double-magic nucleus and that it can be well described by one Slater determinant that contains single-particle wave functions calculated in a mean field approach. Similarly, the nucleus $B$ contains one valence nucleon above double-closed shells and is also described by one Slater determinant. It is also assumed that the single-particle wave functions $\varphi$ in $A$ and $B$ are the same. Furthermore, the center-of-mass motion is neglected. Although this motion is expected to be less important with increasing $A$, it was shown that in the mean field approach its contribution to spectroscopic factors is larger than $1 / A$ [12]. Similar conclusions have been drawn in [13]. With these 
assumptions,

$$
\begin{aligned}
\left\langle\Psi_{M_{B}}^{J_{B}}\right| & \sum_{i \in A} e^{-i \boldsymbol{p} \boldsymbol{x}_{i}}\left|\Psi_{M_{A}}^{J_{A}}\right\rangle \\
= & e^{-i \boldsymbol{p r}}\left\langle\Psi_{M_{B}}^{J_{B}}\left|\sum_{i \in A} e^{i \boldsymbol{p} \boldsymbol{y}_{i}}\right| \Psi_{M_{A}}^{J_{A}}\right\rangle \\
= & \frac{1}{\sqrt{B}} e^{-i \boldsymbol{p r}} \sum_{i}\left[\varphi_{\gamma_{n}}^{*}\left(\boldsymbol{r}_{n}\right)\left\langle\varphi_{\gamma_{i}}\left|e^{i \boldsymbol{p} \boldsymbol{y}_{i}}\right| \varphi_{\gamma_{i}}\right\rangle\right. \\
& \left.-\varphi_{\gamma_{i}}^{*}\left(\boldsymbol{r}_{n}\right)\left\langle\varphi_{\gamma_{n}}\left|e^{i \boldsymbol{p} \boldsymbol{y}_{i}}\right| \varphi_{\gamma_{i}}\right\rangle\right],
\end{aligned}
$$

where $\gamma \equiv\left\{\alpha, m_{j}\right\}, \alpha \equiv\left\{n, l, j, \tau_{j}\right\}$, and $i$ spans all the closedshell orbitals with orbital momentum $l_{i}$, angular momentum $j_{i}$, its projection $m_{i}$, and isospin $\tau_{i}$, while $\gamma_{n}$ denotes the quantum numbers of the (transferred) valence nucleon.

Equation (18) contains contributions from direct and exchange terms. The direct term is equal to

$$
\frac{1}{\sqrt{B}} e^{-i \boldsymbol{p r}} \varphi_{\gamma_{n}}^{*}\left(\boldsymbol{r}_{n}\right) F_{00}(\boldsymbol{p}),
$$

because

$$
\sum_{i}\left\langle\varphi_{\gamma_{i}}\left|e^{i \boldsymbol{p} \boldsymbol{y}_{i}}\right| \varphi_{\gamma_{i}}\right\rangle=F_{00}(\boldsymbol{p}) .
$$

The corresponding direct part of the form factor $\mathcal{S}_{M_{A} M_{B}}^{J_{A} J_{B}}$ is

$$
\mathcal{S}_{M_{A} M_{B}}^{J_{A} J_{B}}(\boldsymbol{q}, \boldsymbol{p})=\frac{1}{\sqrt{B}} \varphi_{\gamma_{n}}^{*}(\boldsymbol{p}-\boldsymbol{q}) F_{00}(\boldsymbol{p})
$$

which is exactly equal to the first term of the expansion in (16) that contains the ground state wave function of nucleus $A$. Therefore, the remaining part of (16), arising due to all possible core excitations, corresponds to nucleon exchanges within the mean field picture of the nuclei.

To calculate the exchange term, one can use the following expression for arbitrary $\gamma_{i}$ and $\gamma_{n}$,

$$
\begin{aligned}
\left\langle\varphi_{\gamma_{n}}\left|e^{i \boldsymbol{p} \boldsymbol{y}}\right| \varphi_{\gamma_{i}}\right\rangle & =\delta_{\tau_{n} \tau_{i}}\left\langle\varphi_{\alpha_{n}}\left|e^{i \boldsymbol{p} \boldsymbol{y}}\right| \varphi_{\alpha_{i}}\right\rangle \\
& =\delta_{\tau_{n} \tau_{i}} \sum_{\lambda \mu} i^{\lambda}\left(\lambda \mu j_{i} m_{i} \mid j_{n} m_{n}\right) \mathcal{F}_{\lambda}^{\alpha_{i} \alpha_{n}}(p) Y_{\lambda \mu}^{*}(\hat{\boldsymbol{p}}),
\end{aligned}
$$

where $\left(\lambda \mu j_{i} m_{i} \mid j_{n} m_{n}\right)$ is the Clebsch-Gordan coefficient, $Y$ is a spherical function, and

$$
\begin{aligned}
\mathcal{F}_{\lambda}^{\alpha_{i} \alpha_{n}}(p)= & \sqrt{4 \pi} \hat{\lambda} \hat{l}_{i} \hat{j}_{i}\left(l_{i} 0 \lambda 0 \mid l_{n} 0\right) W\left(\lambda l_{i} j_{n} \frac{1}{2} ; l_{n} j_{i}\right) \\
& \times \int_{0}^{\infty} d y y^{2} j_{\lambda}(p y) \varphi_{\alpha_{i}}(y) \varphi_{\alpha_{n}}(y) .
\end{aligned}
$$

Here $j_{\lambda}$ is the spherical Bessel function of order $\lambda, W$ is the Racah coefficient, and $\varphi_{\alpha_{i}}\left(r_{n}\right)$ denotes the radial part of the single-particle wave function. Then the contribution from the exchange term is

$$
\begin{aligned}
& \frac{1}{\sqrt{B}} \sum_{i} \varphi_{\gamma_{i}}^{*}\left(\boldsymbol{r}_{n}\right)\left\langle\varphi_{\gamma_{n}}\left|e^{-\boldsymbol{p} \boldsymbol{x}_{i}}\right| \varphi_{\gamma_{i}}\right\rangle \\
& =\frac{1}{\sqrt{B}} \sum_{a \lambda^{\prime \prime} L \alpha_{i}} i^{L} G_{a \lambda^{\prime \prime} L, \mathrm{ex}}^{\alpha_{i} \alpha_{n}}(r, p)\left[\left[Y_{\lambda^{\prime \prime}}^{*}(\hat{\boldsymbol{r}}) \otimes Y_{L}^{*}(\hat{\boldsymbol{p}})\right]_{a} \otimes \phi_{\frac{1}{2}}^{\dagger}\right]_{j_{n} m_{n}},
\end{aligned}
$$

where $\phi_{\frac{1}{2}}$ is the valence neutron spin function and

$$
\begin{aligned}
G_{a \lambda^{\prime \prime} L ; \mathrm{ex}}^{\alpha_{i} \alpha_{n}}(r, p)= & \delta_{\tau_{i} \tau_{n}}(-)^{L+l_{i}-a} \hat{a} \hat{l}_{i} \hat{j}_{i} \varphi_{\alpha_{i}}(r) \sum_{\lambda \lambda^{\prime}}(-)^{\frac{L-\lambda+\lambda^{\prime}}{2}} \hat{\lambda} \hat{\lambda}^{\prime 2} \\
& \times\left(l_{i} 0 \lambda^{\prime} 0 \mid \lambda^{\prime \prime} 0\right)\left(\lambda^{\prime} 0 \lambda 0 \mid L 0\right) W\left(\lambda l_{i} j_{n} \frac{1}{2} ; a j_{i}\right) \\
& \times W\left(L \lambda^{\prime} a l_{i} ; \lambda \lambda^{\prime \prime}\right) j_{\lambda^{\prime}}(p r) \mathcal{F}_{\lambda}^{\alpha_{i} \alpha_{n}}(p) .
\end{aligned}
$$

It is convenient to represent the direct contribution in a form similar to the right-hand side of (24). Then

$$
\begin{aligned}
& \left\langle\Psi_{M_{B}}^{J_{B}}\left|\sum_{i \in A} e^{-i p x_{i}}\right| \Psi_{M_{A}}^{J_{A}}\right\rangle \\
& =\frac{1}{\sqrt{B}} \sum_{a \lambda^{\prime \prime} L \alpha_{i}} i^{L} G_{a \lambda^{\prime \prime} L}^{\alpha_{i} \alpha_{n}}(r, p)\left[\left[Y_{\lambda^{\prime \prime}}^{*}(\hat{\boldsymbol{r}}) \otimes Y_{L}^{*}(\hat{\boldsymbol{p}})\right]_{a} \otimes \phi_{\frac{1}{2}}^{\dagger}\right]_{j_{n} m_{n}},
\end{aligned}
$$

where

$$
G_{a \lambda^{\prime \prime} L}^{\alpha_{i} \alpha_{n}}(r, p)=G_{a \lambda^{\prime \prime} L ; \operatorname{dir}}^{\alpha_{i} \alpha_{n}}(r, p)+G_{a \lambda^{\prime \prime} L ; \mathrm{ex}}^{\alpha_{i} \alpha_{n}}(r, p)
$$

and

$$
G_{a \lambda^{\prime \prime} L ; \operatorname{dir}}^{\alpha_{i} \alpha_{n}}(r, p)=\delta_{a, l_{n}} \hat{j}_{i}^{2} \hat{L}\left(L 0 l_{n} 0 \mid \lambda^{\prime \prime} 0\right) j_{L}(p r) \varphi_{\alpha_{n}}(r) \mathcal{F}_{0}^{\alpha_{i} \alpha_{i}}(p) .
$$

Equations (25)-(28) will be used below to calculate the contribution from the $3 N$ vertex to the $(d, p)$ cross sections.

\section{PWBA AMPLITUDE WITH ZERO-RANGE $3 N$ FORCE}

In this section, $T_{(d, p)}^{\mathrm{PWB}, 3 N}$ is calculated using the simplest possible zero-range $3 N$ force,

$$
W_{i j k}=I_{3} \frac{\delta\left(\boldsymbol{r}_{i j}\right) \delta\left(\boldsymbol{r}_{k i}\right)+\delta\left(\boldsymbol{r}_{j k}\right) \delta\left(\boldsymbol{r}_{i j}\right)+\delta\left(\boldsymbol{r}_{k i}\right) \delta\left(\boldsymbol{r}_{j k}\right)}{3},
$$

where $\boldsymbol{r}_{i j}=\boldsymbol{r}_{i}-\boldsymbol{r}_{j}$ and $I_{3}$ is its volume integral. In this case

$$
D_{M_{p} M_{d}}^{J_{p} J_{d}}(\boldsymbol{k}, \boldsymbol{p}) \equiv \tilde{D}_{M_{p} M_{d}}^{J_{p} J_{d}}=I_{3} \psi_{d}(0) \chi_{J_{p} M_{p}}^{J_{d} M_{d}},
$$

where $\psi_{d}(0)$ is the value of the deuteron wave function at an $n-p$ separation equal to zero, and

$$
\chi_{J_{p} M_{p}}^{J_{d} M_{d}}=\sum_{\sigma_{n}}\left(J_{p} M_{p} \frac{1}{2} \sigma_{n} \mid J_{d} M_{d}\right) \phi_{\frac{1}{2} \sigma_{n}}
$$

is constructed from the transferred neutron spin function $\phi_{\frac{1}{2} \sigma_{n}}$. Potential (29) is similar to the most trivial part of the $3 N$ interaction from the chiral effective field theory (EFT) in next-to-next-to-leading-order (N2LO) $[14,19]$. The latter may contain additional operators $\left(\boldsymbol{\tau}_{j} \cdot \boldsymbol{\tau}_{k}\right)$ that act along with $\delta\left(\boldsymbol{r}_{i j}\right) \delta\left(\boldsymbol{r}_{k i}\right)$. However, in the case of $(d, p)$ reactions including these operators would result in multiplying $\tilde{D}_{M_{p} M_{d}}^{J_{p} J_{d}}$ by an additional operator $\left(\boldsymbol{\tau}_{n} \cdot \boldsymbol{\tau}_{p}\right)+\left(\boldsymbol{\tau}_{n} \cdot \boldsymbol{\tau}_{i}\right)+\left(\boldsymbol{\tau}_{i} \cdot \boldsymbol{\tau}_{p}\right)$, which should act on the product $\chi_{T_{d i} \tau_{d i}}^{(d, i)}$ of deuteron isospin function $\chi_{T_{d} M_{T_{d}}}^{(d)}$ times the isospin function $\chi_{\frac{1}{2} \tau_{i}}$ of nucleon $i$ from $A$. Since deuteron has isospin zero, the isospin of the $d+i$ system is 


\section{$1 / 2$. Since}

$$
\left(\boldsymbol{\tau}_{n} \cdot \boldsymbol{\tau}_{p}\right)+\left(\boldsymbol{\tau}_{n} \cdot \boldsymbol{\tau}_{i}\right)+\left(\boldsymbol{\tau}_{i} \cdot \boldsymbol{\tau}_{p}\right)=2\left(\boldsymbol{T}_{d i}^{2}-\boldsymbol{t}_{n}^{2}-\boldsymbol{t}_{p}^{2}-\boldsymbol{t}_{i}^{2}\right),
$$

where $\boldsymbol{t}_{i}$ is the isospin operator of nucleon $i$ and $\boldsymbol{T}_{d i}$ is the operator of isospin of the $n+p+i$ system, one can conclude that

$$
\left[\left(\boldsymbol{\tau}_{n} \cdot \boldsymbol{\tau}_{p}\right)+\left(\boldsymbol{\tau}_{n} \cdot \boldsymbol{\tau}_{i}\right)+\left(\boldsymbol{\tau}_{i} \cdot \boldsymbol{\tau}_{p}\right)\right] \chi_{\frac{1}{2} \tau_{i}}^{(d, i)}=-\frac{9}{2} \chi_{\frac{1}{2} \tau_{i}}^{(d, i)} .
$$

This means that the $3 N$ force containing isospin operators can effectively be described by (29) in which the volume integral $I_{3}$ is multiplied by the factor of $-9 / 2$.

Since for contact $3 N$ forces $D_{M_{p} M_{d}}^{J_{p} J_{d}}(\boldsymbol{k}, \boldsymbol{p})$ does not depend on any momentum, integration over $d \boldsymbol{p}$ in Eq. (13) can be performed first. This gives

$$
\begin{aligned}
T_{(d, p)}^{\mathrm{ZRPWBA}, 3 N}= & I_{3} \psi_{d}(0) \int d \boldsymbol{r}_{n} e^{i \boldsymbol{q} \boldsymbol{r}_{n}} \\
& \times\left\langle\Psi_{M_{B}}^{J_{B}}\left|\sum_{i \in A} \delta\left(\boldsymbol{r}_{n}-\boldsymbol{y}_{i}\right)\right| \Psi_{M_{A}}^{J_{A}}\right\rangle \chi_{J_{p} M_{p}}^{J_{d} M_{d}} .
\end{aligned}
$$

It is easy to show that, for the particular case of the doublemagic target $A$ considered here, the mean field approach gives

$$
\begin{aligned}
& \left\langle\Psi_{M_{B}}^{J_{B}}\left|\sum_{i \in A} \delta\left(\boldsymbol{r}_{n}-\boldsymbol{y}_{i}\right)\right| \Psi_{M_{A}}^{J_{A}}\right\rangle \\
& =\left\langle\Psi_{M_{B}}^{J_{B}} \mid \Psi_{M_{A}}^{J_{A}}\right\rangle\left[\rho_{A}\left(r_{n}\right)-\frac{1}{2} \rho_{A}^{(\mathrm{n})}\left(r_{n}\right)\right],
\end{aligned}
$$

where the overlap function $\left\langle\Psi_{M_{B}}^{J_{B}} \mid \Psi_{M_{A}}^{J_{A}}\right\rangle$ is just the singleparticle wave function $\varphi_{\gamma_{n}}^{*}\left(\boldsymbol{r}_{n}\right)$ of the valence neutron divided by $\sqrt{B}$, and $\rho_{A}\left(r_{n}\right)$ is the density of target $A$,

$$
\rho_{A}\left(r_{n}\right)=\frac{1}{4 \pi} \sum_{i} \hat{j}_{i}^{2} \varphi_{\alpha_{i}}^{2}\left(r_{n}\right),
$$

while $\rho_{A}^{(\mathrm{n})}\left(r_{n}\right)$ is the neutron component of this density given by Eq. (36), but with the summation carried out over neutron states only. The $T_{(d, p)}^{\mathrm{PWBA}, 3 N}$ has now the same form as $T_{(d, p)}^{\mathrm{PWBA}, 2 N}$ does:

$$
T_{(d, p)}^{\mathrm{ZRPWBA}, 3 N}=\tilde{G}_{M_{B} M_{A}}^{J_{B} J_{A}}(\boldsymbol{q}) \tilde{D}_{M_{p} M_{d}}^{J_{p} J_{d}}
$$

where

$$
\left.\tilde{G}_{M_{B} M_{A}}^{J_{B} J_{A}}(\boldsymbol{q})=\int d \boldsymbol{r}_{n} e^{i \boldsymbol{q} \boldsymbol{r}_{n}}\left|\Psi_{M_{B}}^{J_{B}}\right| \Psi_{M_{A}}^{J_{A}}\right\rangle\left[\rho_{A}\left(r_{n}\right)-\frac{1}{2} \rho_{A}^{(\mathrm{n})}\left(r_{n}\right)\right] .
$$

The corresponding cross section has the same form as well:

$$
\frac{d \sigma^{\mathrm{ZRPWBA}, 3 N}}{d \Omega}=\frac{\mu_{p} \mu_{d}}{4 \pi^{2} \hbar^{4}} \frac{k_{p}}{k_{d}} \frac{\hat{J}_{B}^{2}}{2 \hat{J}_{A}^{2}} \frac{B}{4 \pi}\left(I_{3} \psi_{d}(0)\right)^{2} \tilde{G}_{l_{n} j_{n}}^{2}(q) \text {. }
$$

Equations (37) and (38) can provide a preliminary insight into $3 N$ vertex effects in deuteron stripping, which result in the following qualitatively new features:

(1) The product $\left\langle\Psi_{M_{B}}^{J_{B}} \mid \Psi_{M_{A}}^{J_{A}}\right\rangle \rho_{A}\left(r_{n}\right)$ in Eq. (38) decreases outside the nuclear interior much faster than
$\left\langle\Psi_{M_{B}}^{J_{B}} \mid \Psi_{M_{A}}^{J_{A}}\right\rangle$ does, so that the $3 N$ form factor $\tilde{G}_{M_{B} M_{A}}^{J_{B} J_{A}}(\boldsymbol{q})$ has a shorter range than the $2 N$ form factor does. Therefore, the maximum of the $3 N$ part of the $(d, p)$ cross sections should be shifted towards larger angles with respect to those obtained with the $2 \mathrm{~N}$ vertex only. Therefore, the $3 \mathrm{~N}$ force effects at forward angles, where most measurements are carried out, should be small.

(2) The target excitations due to nucleon exchange result in decreasing the overlap $\left\langle\Psi_{M_{B}}^{J_{B}} \mid \Psi_{M_{A}}^{J_{A}}\right\rangle$ by half the neutron density. This should lead to a reduction of the $3 N$ part of the cross section. For $Z=N$ nuclei, where proton and neutron density distributions are the same, the exchange part, representing the core excitations, gives $25 \%$ contribution to reaction amplitude as compared to the one that comes from the direct term. Therefore, the cross sections obtained with full form factor are 9/16 of those obtained using its direct part only.

(3) Equation (38) suggests that the $3 N$ contribution should be sensitive to asymmetry in the neutron-proton density distributions. Indeed, $(d, p)$ transfers on a neutron-rich target involve the difference of the total target density $\rho_{A}(r)$ and a larger neutron density, which reduces the cross sections coming from the $3 N$ force. However, adding a neutron to a proton-rich target involves subtracting a smaller neutron density from $\rho_{A}(r)$ so that the $3 N$ contribution to the $(d, p)$ cross sections is reduced to a lesser extent. The asymmetry effects may also be sensitive to radial differences in neutron and proton density distributions, in particular, to neutron or proton skins.

These features are investigated numerically in the next section.

\section{PWBA CALCUlations With CONTACT $3 N$ FORCE}

Equation (39) shows that the $3 N$ contribution to the $(d, p)$ cross section is determined by the product of the deuteron wave function $\psi_{d}(0)$, taken at zero separation between neutron and proton, times the volume integral $I_{3}$ of the contact $3 \mathrm{~N}$ force. $\psi_{d}(0)$, being directly determined by the subnucleon short-range physics, is very uncertain. Table I shows its value obtained in several different $N N$ models. They can differ by two orders of magnitude (if the Reid soft core model is excluded). Moreover, the chiral effective field (EFT) at the N4LO predicts a node in the short-range part of the deuteron wave function when small regulators are used [18], making $\psi_{d}(0)$ negative, with consequences for interference between $2 N$ and $3 N$ contributions. The $3 N$ force model parameters are usually fitted to reproduce some observables calculated for a fixed $2 \mathrm{~N}$ model and, therefore, the volume integral $I_{3}$ should be consistent with the model for $\psi_{d}$. In this paper, $\psi_{d}(0)$ and $I_{3}$ are taken from Lynn et al. [19], in which the $3 \mathrm{~N}$ interactions consisted of regularized contact and twopion exchange terms of different operator formats, and the strengths of these terms $\left(c_{E}\right.$ and $c_{D}$, respectively) were fitted to simultaneously reproduce light nuclei $(A=3,4,5)$ binding 
TABLE I. Deuteron wave function at zero $n-p$ separations (in $\mathrm{fm}^{-3 / 2}$ ) for the different $N N$-model interactions introduced in the references shown. The chiral $(\chi)$ EFT wave functions use the different regulators shown.

\begin{tabular}{lcc}
\hline \hline$N N$ model & Ref. & $\psi_{d}(0) / Y_{00}(\hat{\boldsymbol{r}})$ \\
\hline Reid soft core & {$[15]$} & 0 \\
Argonne V18 & {$[16]$} & 0.079 \\
CD-Bonn & {$[17]$} & 0.30 \\
$\chi$ EFT N4LO: $0.8 \mathrm{fm}$ & {$[18]$} & -0.22 \\
$\chi$ EFT N4LO: $0.9 \mathrm{fm}$ & {$[18]$} & -0.11 \\
$\chi$ EFT N4LO: $1.0 \mathrm{fm}$ & {$[18]$} & -0.026 \\
$\chi$ EFT N4LO: $1.1 \mathrm{fm}$ & {$[18]$} & 0.062 \\
$\chi$ EFT N4LO: $1.2 \mathrm{fm}$ & {$[18]$} & 0.14 \\
$\chi$ EFT N2LO: $1.0 \mathrm{fm}$ & {$[19]$} & 0.282 \\
\hline \hline
\end{tabular}

energies, neutron- $\alpha$ scattering, and neutron matter properties within chiral EFT at N2LO. The recommended values of $c_{E}$ and $c_{D}$ are summarized in Table I of [19], and three out of five pairs of these values correspond to $c_{D}=0$, the case without two-pion exchange. The volume integral $I_{3}$, used in the present paper, is calculated with the interaction parameters from the first row of Table I of [19], corresponding to the $\left(\tau_{i} \cdot \tau_{k}\right)$ operator format and no $2 \pi$ exchange. In this case

$$
I_{3}=-\frac{9}{2} \frac{c_{E}}{F_{\pi}^{4} \Lambda_{\chi}},
$$

where $c_{E}=-0.63$ is the low-energy constant, $F_{\pi}=$ 92.4 $\mathrm{MeV}$ is the weak pion decay constant, and $\Lambda_{\chi}=$ $700 \mathrm{MeV}$ is chiral symmetry breaking scale, leading to $I_{3}=$ $3280 \mathrm{MeV} \mathrm{fm}^{6}$. The EFT deuteron wave function at N2LO is $\psi_{d}(0)=0.282 / \sqrt{4 \pi}$.

With the chosen values of $I_{3}$ and $\psi_{d}(0)$, the PWBA calculations have been performed for two double-magic targets, ${ }^{40} \mathrm{Ca}$ and ${ }^{208} \mathrm{~Pb}$, using single-particle wave functions from spherical Hartree-Fock calculations with the Skyrm SkP interaction [20]. The single-particle energies of valence nucleons are reproduced within $1.5 \mathrm{MeV}$, which is sufficient for the purposes of this paper. Two deuteron incident energies, 10 and $100 \mathrm{MeV}$, have been chosen, which represent the the lowest (TRUIMF) and the highest (RIKEN) ends of the energy range available in radioactive beam facilities worldwide. To illustrate asymmetry in core excitation effects, $(d, n)$ calculations have been done as well. The analytical expressions for $(d, n)$ reactions are identical to those discussed above, except for neutron density $\rho_{A}^{(\mathrm{n})}$ in Eq. (38) replaced by proton density $\rho_{A}^{(\mathrm{p})}$. For the $N=Z$ nucleus ${ }^{40} \mathrm{Ca}$, the core excitation contributions should be the same both for $(d, p)$ and $(d, n)$ reactions, while for ${ }^{208} \mathrm{~Pb}$ their relative contributions should differ. This is demonstrated in Fig. 2, where the ratios of differential PWBA cross sections calculated at $E_{d}=10 \mathrm{MeV}$ with direct form factor only to those obtained with full form factor are plotted as a function of scattering angle. For the ${ }^{40} \mathrm{Ca}$ target, this ratio is close to $16 / 9$, for both $(d, p)$ and $(d, n)$ reactions, while for ${ }^{208} \mathrm{~Pb}$ it displays asymmetry. For $E_{d}=100 \mathrm{MeV}$, the cross sections, calculated with direct only and total form factors, have more

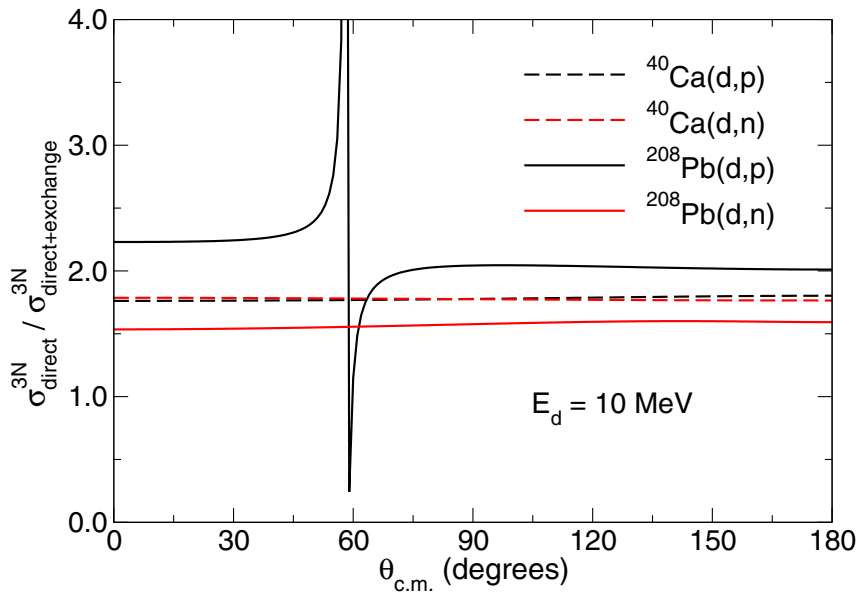

FIG. 2. The ratio of $3 N$ PWBA differential cross sections calculated with the direct form factor only to those obtained with the full form factor for ${ }^{40} \mathrm{Ca}(d, p){ }^{41} \mathrm{Ca},{ }^{40} \mathrm{Ca}(d, n){ }^{41} \mathrm{Sc},{ }^{208} \mathrm{~Pb}(d, p){ }^{209} \mathrm{~Pb}$, and ${ }^{208} \mathrm{~Pb}(d, n){ }^{209} \mathrm{Bi}$ reactions at $E_{d}=10 \mathrm{MeV}$.

oscillations with non-coinciding minima. For this reason, it is more difficult to illustrate the $n$ - $p$ asymmetry.

Figure 3 compares the PWBA cross sections calculated with $2 N$ or $3 N$ forces only. Full form factors were used in the $3 N$ case. The calculations with the $2 N$ force include the deuteron $s$ state only, and are practically insensitive to the choice of the model for the vertex form factor $D_{M_{p} M_{d}}^{J_{p} J_{d}}(k)$. For consistency, this form factor is calculated using a chiral EFT N2LO deuteron wave function. The figure shows that $3 N$ vertex effects are very small at very forward angles: less than $1 \%$. They increase with scattering angle and become competitive to the $2 \mathrm{~N}$ cross sections where the latter decrease significantly. Generally, the importance of $3 N$ contribution increases with deuteron incident energy.

\section{INCLUDING DISTORTIONS}

In the case of a contact $3 N$ force, distortions in incident and exit channels of a $(d, p)$ reaction can be easily included. Distorted waves can be represented by the plane-wave expansions [10]. For each chosen plane wave, the $3 N$ part of the $(d, p)$ amplitude contains a modification of the overlap integral, given by Eq. (35), which is independent of any momentum. If the $2 N$ part is also treated in the zero-range approximation, which assumes $V_{n p} \psi_{d} \approx D_{0} \delta\left(\boldsymbol{r}_{n}-\boldsymbol{r}_{p}\right)$ and, therefore,

$$
D_{J_{d} M_{d}}^{J_{p} M_{p}}(\boldsymbol{k}) \approx D_{0} \chi_{J_{d} M_{d}}^{J_{p} M_{p}}
$$

then the sum $T_{(d, p)}^{\mathrm{PW}}$ of the $2 N$ and $3 N$ transitions between any plane waves is

$$
\begin{aligned}
T_{(d, p)}^{\mathrm{PW}} & =\left(D_{0} G_{M_{B} M_{A}}^{J_{B} J_{A}}(\boldsymbol{q})+I_{3} \psi_{d}(0) \tilde{G}_{M_{B} M_{A}}^{J_{B} J_{A}}(\boldsymbol{q})\right) \chi_{J_{d} M_{d}}^{J_{p} M_{p}} \\
& =D_{0} \int d \boldsymbol{r}_{n} e^{i \boldsymbol{q} \boldsymbol{r}_{n}} I_{J_{B} M_{B} J_{A} M_{A}}^{\mathrm{mod}}\left(\boldsymbol{r}_{n}\right) \chi_{J_{d} M_{d}}^{J_{p} M_{p}}
\end{aligned}
$$



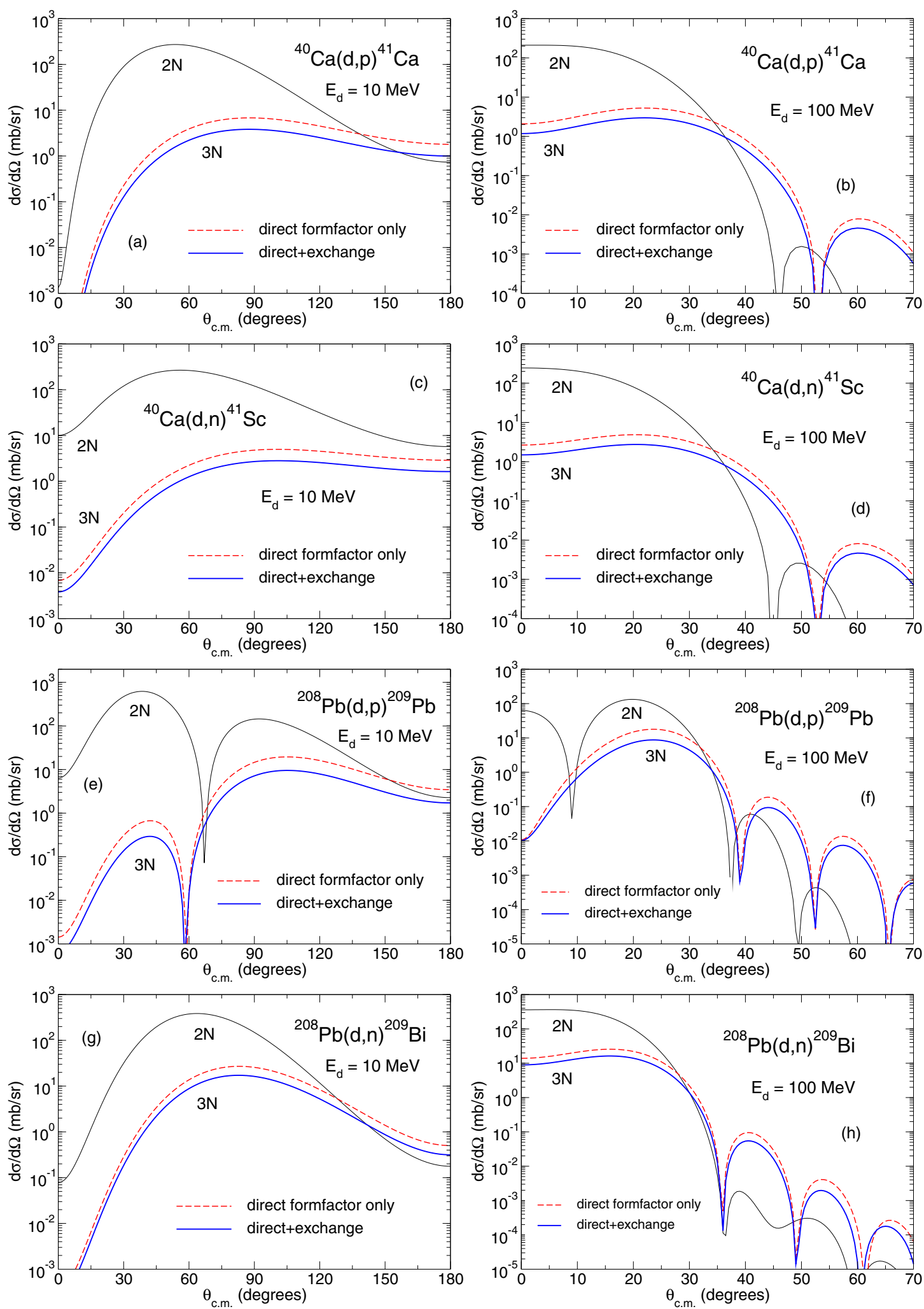

FIG. 3. The PWBA differential cross sections of ${ }^{40} \mathrm{Ca}(d, p){ }^{41} \mathrm{Ca}(a, b), \quad{ }^{40} \mathrm{Ca}(d, n){ }^{41} \mathrm{Sc}(c, d), \quad{ }^{208} \mathrm{~Pb}(d, p){ }^{209} \mathrm{~Pb}(e, f), \quad$ and ${ }^{208} \mathrm{~Pb}(d, n){ }^{209} \mathrm{Bi}(g, h)$ reactions corresponding contributions from zero-range $3 N$ force in comparison with that calculated with $2 N$ force only. Two incident deuteron laboratory energies are used: $10 \mathrm{MeV}$ [(a), (c), (e), (g)] and $100 \mathrm{MeV}$ [(b), (d), (f), (h)]. 
where

$$
\begin{aligned}
& I_{J_{B} M_{B} J_{A} M_{A}}^{\bmod }(\boldsymbol{r}) \\
& \quad=\left\langle\Psi_{M_{B}}^{J_{B}} \mid \Psi_{M_{A}}^{J_{A}}\right\rangle\left[1+\frac{I_{3} \psi_{d}(0)}{D_{0}}\left(\rho_{A}(r)-\frac{1}{2} \rho_{A}^{(\mathrm{n})}(r)\right)\right] .
\end{aligned}
$$

Therefore, performing summation over plane waves back will give us the standard zero-range distorted-wave $(d, p)$ reaction model but with a modified overlap function. The corresponding cross sections can be calculated using any reaction code that allows overlap functions to be read in.

The influence of the $3 N$ vertex beyond the PWBA has been investigated here for ${ }^{40} \mathrm{Ca}(d, p){ }^{41} \mathrm{Ca}$ and ${ }^{208} \mathrm{~Pb}(d, p){ }^{209} \mathrm{~Pb}$ at two incident deuteron energies, 10 and $100 \mathrm{MeV}$, in both DWBA and ADWA. In DWBA, global optical potentials from [21] and [22] were used to generate distorted waves in the incident deuteron and exit proton channels, respectively. In ADWA, the deuteron distorting potential was constructed according to the Johnson-Soper prescription [23] using the same Chapel-Hill systematics [22] for proton-target and neutrontarget optical potentials. In both calculations the spherical Hartree-Fock single-particle wave function from the previous section was used to represent the overlap function $\langle B \mid A\rangle$, which then has been modified by the factor containing HartreeFock densities according to Eq. (43). The modified overlap has been read into TWOFNR [24]. In these calculations the zero-range constant $D_{0}=-15903.73 \mathrm{MeV} \mathrm{fm}^{3 / 2}$ was used together with the same values of $I_{3}$ and $\psi_{d}(0)$ as in the plane-wave calculations.

Distortions of scattering waves in deuteron and proton channels have profound effects on the shape of the $(d, p)$ cross sections calculated with the $3 N$ force only. This can be seen from comparison of the $3 N$ PWBA cross sections of Fig. 3 with the corresponding DWBA and ADWA cross sections in Fig. 4. The maximum of the latter moves towards smaller angles, and their angular spread becomes narrower. This could be qualitatively understood by realizing that due to the modification of the overlap function most of the contribution to the $3 \mathrm{~N}$ cross section comes from the nuclear interior. In the internal region, the local kinetic energy of scattering particles is larger because of negative values of the nuclear potential. Therefore, in the plane-wave expansion of the distorted-wave amplitude (5), the plane waves with higher incoming energy will be preferably selected. The cross sections at higher incident energies are always peaked at smaller angles. As a result, the distorted-wave cross sections calculated with $2 N$ and $3 N$ forces only have much less difference than those in the PWBA case have, and the influence of the $3 N$ effects at small angles becomes more important. This can be seen in Fig. 4.

In general, the $3 N$ effects affect the $(d, p)$ cross sections in a different way depending on the choice of the distorted-wave theory, which is explained by different contributions from the internal nuclear area in DWBA and ADWA. The $3 \mathrm{~N}$ contribution also depends on the deuteron energy. It is 4-7\% at $E_{d}=10 \mathrm{MeV}$ increasing to $15 \%$ for $E_{d}=100 \mathrm{MeV}$ for the ${ }^{40} \mathrm{Ca}$ target. For ${ }^{208} \mathrm{~Pb}$, this contribution is even larger: $25 \%$ in the DWBA. The ${ }^{208} \mathrm{~Pb}(d, p){ }^{209} \mathrm{~Pb}$ reaction at $10 \mathrm{MeV}$ is a special case. At this energy the Coulomb barrier shifts the $2 N$ cross sections towards back angles. However, the effective incoming energy, required in the $3 N$ case, is of the order of the nucleon ${ }^{208} \mathrm{~Pb}$ potential well, which is larger than the Coulomb barrier of $\sim 20 \mathrm{MeV}$, so that the corresponding $(d, p)$ cross sections are peaked at small angles. As a result, no $3 N$ contributions are expected in the maxima of the sub-Coulomb $(d, p)$ reactions.

\section{FINITE-RANGE $3 N$ FORCE}

The contact $3 N$ force selects only one value of the deuteron wave function to contribute to the $(d, p)$ amplitude. This value, $\psi_{d}(0)$, taken at zero $n$ - $p$ separation $r$, is small due to repulsive nature of the $N N$ force core, which is seen in all realistic $N N$ models. One can expect that a finite-range $3 N$ force will select a larger range of $r$, where the strength of the repulsive core decreases and the corresponding $\psi_{d}(r)$ may be larger. This should increase the $(d, p)$ cross sections. Incorporating finite-range effects properly requires new development efforts in distorted-wave theories at both formal and coding levels. Therefore, in this section finite-range effects are estimated within the PWBA.

A finite-range $3 N$ force corresponds to some regularization of the contact N2LO interaction. Here, the simplest possible force is considered: the hypercentral Gaussian force of range $\rho_{0}$. Usually, the radial dependence of regulators used in $3 N$ models is steeper. For example, in [19] they have been chosen to decrease with $r$ as $\exp \left[-(r / R)^{4}\right]$, and the $c_{E}=-0.63$ value used in previous sections corresponds to $R=1.0 \mathrm{fm}$. However, using a smoother regulator is justified for the first study of the finite-range $3 N$ effects in $(d, p)$ reactions. In this study, the range is allowed to vary while the volume integral of this force remains the same as in previous sections. The $3 N$ force is defined as

$$
W_{i j k} \equiv W\left(\rho_{i j k}\right)=W_{0} e^{-\frac{\rho_{i j k}^{2}}{\rho_{0}^{2}}}=W_{0} e^{-\frac{1}{3} \frac{r_{i j}^{2}+r_{j k}^{2}+r_{k i}^{2}}{\rho_{0}^{2}}},
$$

where $\rho_{i j k}$ is the hyperradius. Its strength $W_{0}$ is related to the volume integral $I_{3}$ by equation $I_{3}=3^{3 / 2} \pi^{3} W_{0} \rho_{0}^{6}$. Here, $I_{3}$ is defined as

$$
I_{3}=\int d \boldsymbol{r}_{12} d \boldsymbol{r}_{13} W\left(\rho_{i j k}\right),
$$

to be consistent with the zero-range definition. For this hypercentral force, Eq. (14) becomes

$$
D_{M_{p} M_{d}}^{J_{p} J_{d}}(\boldsymbol{k}, \boldsymbol{p})=f(\boldsymbol{p}) g\left(\boldsymbol{k}+\frac{1}{2} \boldsymbol{p}\right) \chi_{\sigma_{p}}^{J_{d} M_{d}}
$$

where

$$
\begin{aligned}
f(\boldsymbol{p}) & =W_{0} \int d \boldsymbol{x} e^{i \boldsymbol{p} \boldsymbol{x}} e^{-\frac{2}{3} \frac{x^{2}}{\rho_{0}^{2}}}=\frac{I_{3}}{(2 \pi)^{3 / 2} \rho_{0}^{3}} e^{-\frac{3}{8} p^{2} \rho_{0}^{2}}, \\
g(\boldsymbol{p}) & =\int d \boldsymbol{r} e^{i \boldsymbol{p} \boldsymbol{r}} e^{-\frac{1}{2} \frac{r^{2}}{\rho_{0}^{2}}} \psi_{d}(\boldsymbol{r}) \\
& =\sqrt{4 \pi} \int_{0}^{\infty} d r r^{2} j_{0}(p r) e^{-\frac{1}{2} \frac{r^{2}}{\rho_{0}^{2}}} \varphi_{d}^{(s)}(r),
\end{aligned}
$$

and $\varphi_{d}^{(s)}(r)$ is the $s$-wave part of the deuteron wave function. The $d$-wave part is neglected. To use $D_{M_{p} M_{d}}^{J_{p} J_{d}}(\boldsymbol{k}, \boldsymbol{p})$ in the PWBA amplitude (13), the partial wave expansion of $g\left(\boldsymbol{k}+\frac{1}{2} \boldsymbol{p}\right)$ is 

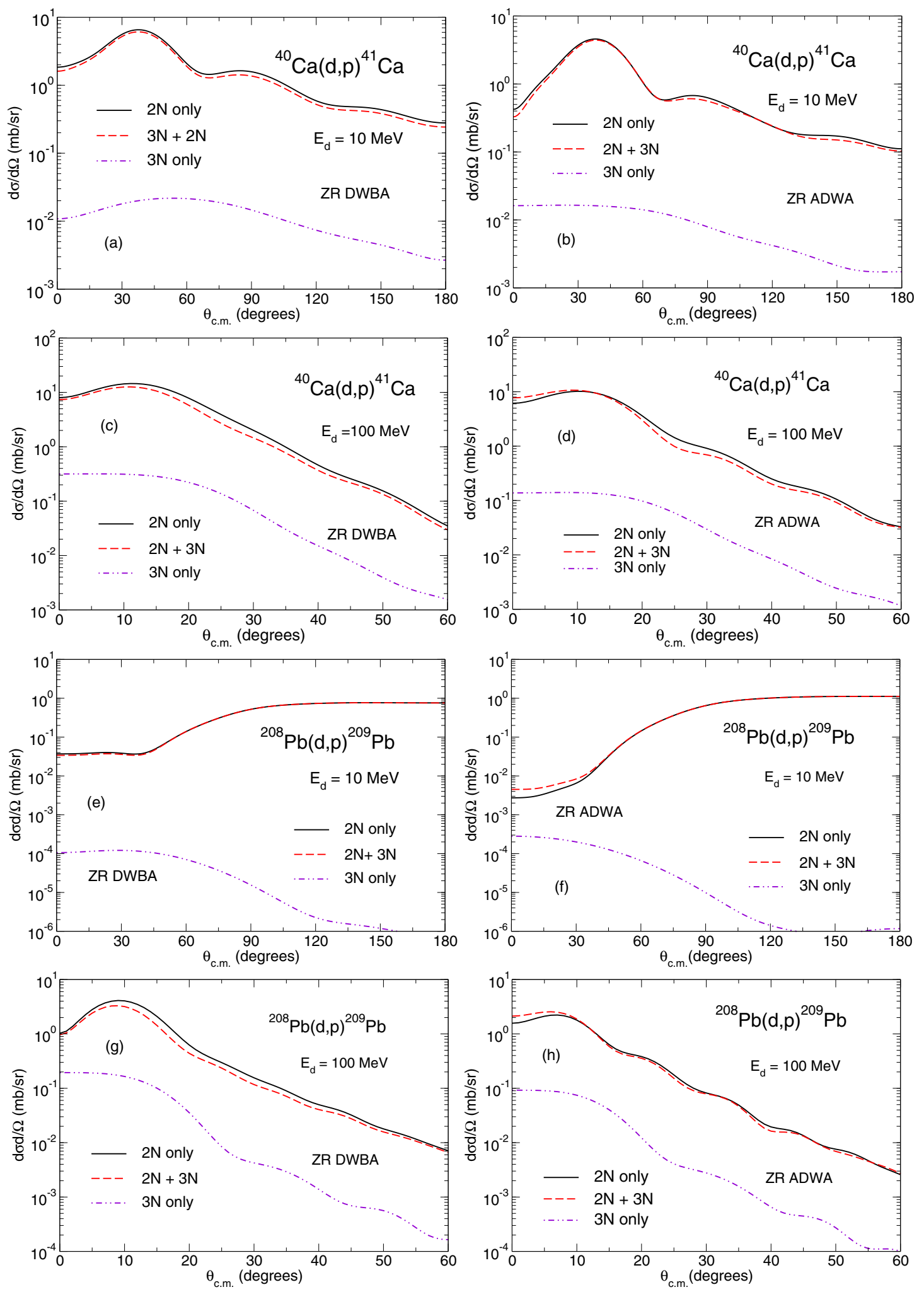

FIG. 4. The DWBA [(a), (c), (e), (g)] and ADWA [(b), (d), (f), (h)] differential cross sections of ${ }^{40} \mathrm{Ca}(d, p){ }^{41} \mathrm{Ca}(\mathrm{a})-(\mathrm{d})$ and ${ }^{208} \mathrm{~Pb}(d, p){ }^{209} \mathrm{~Pb}$ (e)-(h) reactions calculated with $2 N$ force only and with $2 N$ force plus contact $3 N$ force. Two incident deuteron laboratory energies are used: $10 \mathrm{MeV}$ [(a), (b), (e), (f)] and $100 \mathrm{MeV}$ [(c), (d), (g), (h)]. 
introduced:

$$
\begin{aligned}
g\left(\boldsymbol{k}+\frac{1}{2} \boldsymbol{p}\right) & =4 \pi \sum_{\lambda \mu}(-)^{\lambda} g_{\lambda}\left(k, \frac{1}{2} p\right) Y_{\lambda \mu}^{*}(\hat{\boldsymbol{k}}) Y_{\lambda \mu}(\hat{\boldsymbol{p}}), \\
g_{\lambda}(k, p) & =\frac{1}{2} \int_{-1}^{1} g\left(\left|\boldsymbol{k}+\frac{1}{2} \boldsymbol{p}\right|\right) P_{\lambda}\left(\cos \theta_{k p}\right) d\left(\cos _{k p}\right),
\end{aligned}
$$

where $\theta_{k p}$ is the angle between vectors $\boldsymbol{k}$ and $\boldsymbol{p}$ and

$$
\cos \theta_{k q}=\frac{\frac{1}{2} k_{d}^{2}-k_{d} k_{p} \cos \theta+\frac{A}{A+1} k_{p}^{2}}{k q} .
$$

With these expressions and the mean field results (25), (26), (27), and (28) for the nuclear form factor, obtained for doubleclosed-shell targets in Sec. IV, the $3 N$ contribution to the $(d, p)$ amplitude becomes

$$
\begin{aligned}
T_{(d, p)}^{\mathrm{PWBA}, 3 N}= & \sum_{a m_{a} \sigma_{n}}\left(a m_{a} \frac{1}{2} \sigma_{n} \mid j_{n} m_{n}\right) \\
& \times\left(J_{p} M_{p} \frac{1}{2} \sigma_{n} \mid J_{d} M_{d}\right) T_{a m_{a}}(\boldsymbol{k}, \boldsymbol{q}),
\end{aligned}
$$

where

$$
\begin{aligned}
T_{a m_{a}}(\boldsymbol{k}, \boldsymbol{q})= & \frac{1}{(2 \pi)^{3}} \int d \boldsymbol{r} e^{i \boldsymbol{q} \boldsymbol{r}} \int d \boldsymbol{p} f(p) g\left(\boldsymbol{k}+\frac{1}{2} \boldsymbol{p}\right) \\
& \times\left\langle\Psi_{M_{B}}^{J_{B}}\left|\sum_{i \in A} e^{i \boldsymbol{p} \boldsymbol{x}_{i}}\right| \Psi_{M_{A}}^{J_{A}}\right\rangle \\
= & \frac{2}{\pi \sqrt{B}} \sum_{\lambda^{\prime \prime L}} i^{\lambda^{\prime \prime}-L} T_{a \lambda^{\prime \prime} L}^{\alpha_{n}}(k, q)\left[Y_{\lambda^{\prime \prime}}^{*}(\hat{\boldsymbol{q}}) \otimes Y_{L}^{*}(\hat{\boldsymbol{k}})\right]_{a m_{a}}
\end{aligned}
$$

and

$$
\begin{aligned}
T_{a \lambda^{\prime \prime} L}^{\alpha_{n}}(k, q)= & \int_{0}^{\infty} d r r^{2} j_{\lambda^{\prime \prime}}(q r) \int_{0}^{\infty} d p p^{2} f(p) \\
& \times g_{L}\left(k, \frac{1}{2} p\right) \sum_{\alpha_{i}} G_{a \lambda^{\prime \prime} L}^{\alpha_{i} \alpha_{n}}(r, p) .
\end{aligned}
$$

The $2 N$ part of the $(d, p)$ amplitude, written in a similar way,

$$
T_{a \lambda^{\prime \prime} L}^{\alpha_{n}, 2 N}(k, q)=\delta_{L, 0} \delta_{a, l_{n}} \frac{D(k)}{\pi^{3 / 2} \sqrt{B}},
$$

could be added to (54) if calculations of interference between $2 N$ and $3 N$ transfer amplitudes are required.

The cross section, corresponding to the amplitude represented by Eq. (52), is

$$
\begin{aligned}
\frac{d \sigma}{d \Omega}= & \frac{\mu_{p} \mu_{d}}{4 \pi^{2} \hbar^{4}} \frac{k_{p}}{k_{d}} \frac{B}{\hat{J}_{d}^{2} \hat{J}_{A}^{2}} \sum_{M_{d} M_{p} M_{A} M_{B}}\left|T_{(d, p)}\right|^{2} \\
= & \frac{\mu_{p} \mu_{d}}{4 \pi^{2} \hbar^{4}} \frac{k_{p}}{k_{d}} \frac{\hat{j}_{n}^{2}}{2} \sum_{a \lambda^{\prime \prime} \tilde{\lambda}^{\prime \prime} L \tilde{L}} T_{a \lambda^{\prime \prime} L}^{\alpha_{n}}(k, q) T_{a \tilde{\lambda}^{\prime \prime} \tilde{L}}^{\alpha_{n}}(k, q) \\
& \times \Theta_{a}^{\lambda^{\prime \prime} L \tilde{\lambda}^{\prime \prime} \tilde{L}}\left(\cos \theta_{k q}\right),
\end{aligned}
$$
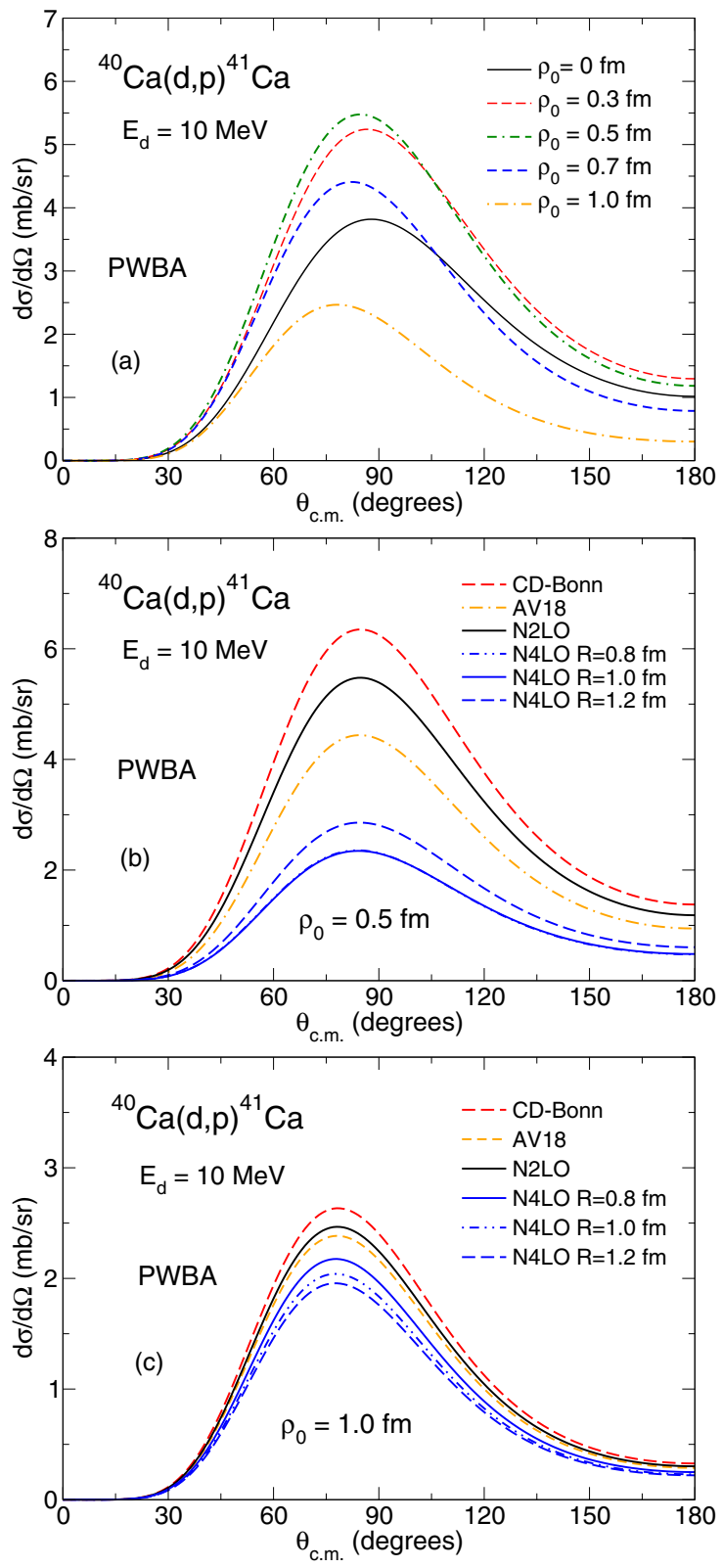

FIG. 5. The ${ }^{40} \mathrm{Ca}(d, p){ }^{41} \mathrm{Ca}$ cross sections calculated at $E_{d}=$ $10 \mathrm{MeV}$ using (a) several ranges of the three-body force and the deuteron wave function from chiral EFT at N2LO, and using several deuteron wave functions and the range parameters $\rho_{0}=0.5 \mathrm{fm}(\mathrm{b})$ and $\rho_{0}=1.0 \mathrm{fm}$ (c).

where

$$
\begin{aligned}
& \Theta_{a}^{\lambda^{\prime \prime} L \tilde{\lambda}^{\prime \prime} \tilde{L}}\left(\cos \theta_{k q}\right) \\
& =(-)^{\frac{\lambda^{\prime \prime}-L \tilde{\lambda}^{\prime \prime}+\tilde{L}}{2}+a} \frac{\hat{\lambda}^{\prime \prime} \hat{\tilde{\lambda}}{ }^{\prime \prime} \hat{L} \hat{\tilde{L}}}{4 \pi^{4}} \sum_{b}\left(\lambda^{\prime \prime} 0 \tilde{\lambda}^{\prime \prime} 0 \mid b 0\right)(L 0 \tilde{L} 0 \mid b 0) \\
& \quad \times W\left(\lambda^{\prime \prime} L \tilde{\lambda} \tilde{\lambda}^{\prime \prime} \tilde{L} ; a b\right) P_{b}\left(\cos \theta_{k q}\right),
\end{aligned}
$$

and $P$ is the Legendre polynomial. In the $\rho_{0} \rightarrow 0$ limit the cross section (56) reduces to that given by Eq. (39).

Numerical calculations have been performed with the same volume integral $I_{3}=3280 \mathrm{MeV} \mathrm{fm}^{6}$ as in Sec. VI, and are 


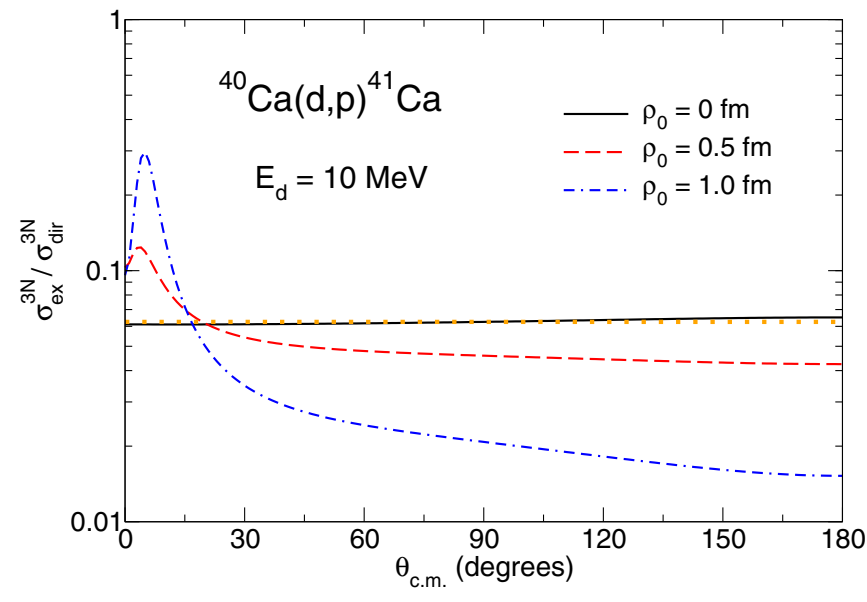

FIG. 6. The ratio of $3 N$ PWBA differential cross sections calculated with the exchange form factor only to those obtained with the direct form factor for the ${ }^{40} \mathrm{Ca}(d, p){ }^{41} \mathrm{Ca}$ reaction at $E_{d}=10 \mathrm{MeV}$ using the N2LO deuteron wave function and several ranges $\rho_{0}$ of the hypercentral three-body force. The dots correspond to the constant $1 / 16$, which is expected for the zero-range result obtained with identical neutron and proton densities.

presented for the $3 N$ contribution only. No interference with the $2 N$ term has been calculated because, as shown in the previous section, distortions of scattering proton and deuteron waves can completely change it. Figure 5 shows the $3 \mathrm{~N}$ contributions to ${ }^{40} \mathrm{Ca}(d, p){ }^{41} \mathrm{Ca}$ cross sections calculated for $E_{d}=10 \mathrm{MeV}$ with full nuclear form factors and several values of $\rho_{0}$, keeping the chiral EFT N2LO model for $\psi_{d}$. One can see that with increasing $\rho_{0}$ the cross sections at first increase but then decrease again [Fig. 5(a)]. This happens because there exist some $\rho_{0}$ for which overlap between the $3 N$ force $W_{n p i}$ and the deuteron wave function $\psi_{d}$ is optimal. Selecting $\rho_{0}=0.5 \mathrm{fm}$ for which the cross sections are the largest, calculations with several deuteron model wave functions have been done [see Fig. 5(b)]. The calculations reveal a large spread of the cross sections, within a factor of 3 , that are largest for the CD-Bonn deuteron wave function and smallest for the chiral EFT at N4LO with regulators of 1.0 and $1.2 \mathrm{fm}$. However, at a larger value of $\rho_{0}$ the spread between the cross sections, obtained with different deuteron wave functions, gets smaller [see Fig. 5(c)]. This is not surprising because with larger $\rho_{0}$ the overlap between $W_{n p i}$ and $\psi_{d}$ is determined by larger distances between the neutron and proton in the deuteron, where all $N N$ models predict the same behavior for $\psi_{d}$.

Finally, the relative contributions from direct and exchange form factors for a finite-range $3 N$ force have also been investigated. Figure 6 shows the ratio of the exchange to direct PWBA cross sections of ${ }^{40} \mathrm{Ca}(d, p){ }^{41} \mathrm{Ca}$ at $E_{d}=10 \mathrm{MeV}$, calculated for $\rho_{0}=0,0.5$, and $1.0 \mathrm{fm}$. For a contact force, this ratio is practically independent of the scattering angle and is close to $1 / 16$, as explained in Secs. V and VI. However, with increasing $\rho_{0}$ this ratio increases at small angles and decreases at large angles. This trend will most likely persist when distortions in deuteron and proton channels are included.

\section{SUMMARY AND CONCLUSION}

Within phenomenology-oriented distorted-wave $(d, p)$ reaction theories, the $3 N$ force effects are implicitly included in optical potentials, fitted to experimental elastic scattering data, and overlap integrals. However, a nontrivial contribution should come from a $3 N$ interaction between the neutron and proton in the incoming deuteron with one of the nucleons in the target. The present paper has examined the role of this term for a simple hypercentral form of the $3 N$ force.

It has been shown that the $3 N$ contribution to the $A(d, p) B$ amplitude contains a new matrix element (form factor) which contains the overlap between the wave functon of nucleus $B$ with all excited state wave functions of the target $A$, thus invalidating exact factorization of one-step $(d, p)$ cross sections in terms of spectroscopic factors. The calculations of the new form factor have been done using the Hartree-Fock wave functions, assuming that the target is a double-closed-shell nucleus. In this case, all target excitations are taken into account via nucleon exchanges.

It has also been shown that for the contact $3 N$ interaction the $A(d, p) B$ amplitude takes the standard form, except for the overlap function $\langle A \mid B\rangle$ being modified in a simple way. This allows available reaction codes to be used to estimate the zero-range $3 N$ effects. With the volume integral of the contact force compatible with the latest study of light nuclei, $\alpha+N$ scattering, and nuclear matter [19] and with the chiral EFT deuteron wave function at N2LO, compatible with this force, the $(d, p)$ cross sections on ${ }^{40} \mathrm{Ca}$ and ${ }^{208} \mathrm{~Pb}$ targets were calculated for incident deuteron energies of 10 and $100 \mathrm{MeV}$. The contribution of the $3 N$ term for energies above the Coulomb barrier is noticeable: up to $7 \%$ at $10 \mathrm{MeV}$ and up to $25 \%$ ar $100 \mathrm{MeV}$. This is a relatively large contribution but it can be strongly affected by the finite range of the $3 N$ force.

At present, finite-range $3 N$ effects have been calculated in the PWBA only, as distorted-wave calculations involve the angular momentum structure of the $3 \mathrm{~N}$ form factor and its additional dependence on a target nucleon momentum, which are incompatible with the formal structure of the transfer $T$ matrix used in available reactions codes. Development of new distorted-wave codes would be justified if a preliminary assessment of the $3 \mathrm{~N}$ contribution showed their important role. This seems to be the case for high deuteron incident energies, and that is where such developments may prove to be beneficial.

Finally, when most of the present calculations were finished, Minomo et al. published their investigation of $3 N$ effects in $(p, 2 p)$ reactions at high energies within the distorted-wave impulse approximation [25]. They came to the conclusion that the $3 N$ contribution is small in most cases apart from some of those with special kinematic conditions for knockout of protons from deeply bound orbits. These conditions could be used to study the $3 N$ force effects experimentally.

\section{ACKNOWLEDGMENTS}

I am grateful to J. E. Lynn for providing deuteron wave function from chiral EFT at N2LO and E. Epelbaum for providing deuteron wave functions from chiral EFT at N4LO. 
I am also grateful to P. D. Stevenson for providing me with his Hartree-Fock code. This work was supported by the United
Kingdom Science and Technology Facilities Council (STFC) under Grant No. ST/L005743/1.
[1] A. E. Lovell and F. M. Nunes, J. Phys. G 42, 034014 (2015).

[2] G. W. Bailey, N. K. Timofeyuk, and J. A. Tostevin, Phys. Rev. Lett. 117, 162502 (2016); 119, 159901(E) (2017).

[3] G. W. Bailey, N. K. Timofeyuk, and J. A. Tostevin, Phys. Rev. C 95, 024603 (2017).

[4] N. Austern, Direct Nuclear Reaction Theories (Wiley, New York, 1970).

[5] G. R. Satchler, Direct Nuclear Reactions (Oxford University Press, New York, 1983).

[6] R. C. Johnson and P. C. Tandy, Nucl. Phys. A 235, 56 (1974).

[7] R. C. Johnson and N. K. Timofeyuk, Phys. Rev. C 89, 024605 (2014).

[8] M. L. Goldberger and K. M. Watson, Collision Theory (Dover, New York, 2004).

[9] N. K. Timofeyuk and R. C. Johnson, Phys. Rev. C 59, 1545 (1999).

[10] L. A. Charlton, Phys. Rev. C 8, 146 (1973).

[11] P. D. Kunz, DWUCK5 code, http://spot.colorado.edu/ kunz/ DWBA.html

[12] R. R. Rodriguez-Guzman and K. W. Schmid, Eur. Phys. J. A 19, 61 (2004).
[13] N. K. Timofeyuk, Phys. Rev. C 84, 054313 (2011).

[14] P. Navratil, Few-Body Syst. 41, 117 (2007).

[15] R. V. Reid, Ann. Phys. (N.Y.) 50, 411 (1968).

[16] R. B. Wiringa, V. G. J. Stoks, and R. Schiavilla, Phys. Rev. C 51, 38 (1995).

[17] R. Machleidt, Phys. Rev. C 63, 024001 (2001).

[18] E. Epelbaum, H. Krebs, and U.-G. Meißner, Phys. Rev. Lett. 115, 122301 (2015).

[19] J. E. Lynn, I. Tews, J. Carlson, S. Gandolfi, A. Gezerlis, K. E. Schmidt, and A. Schwenk, Phys. Rev. Lett. 116, 062501 (2016).

[20] J. Dobaczewski, H. Flocard, and J. Treiner, Nucl. Phys. A 422, 103 (1984).

[21] W. W. Daehnick, J. D. Childs, and Z. Vrcelj, Phys. Rev. C 21, 2253 (1980).

[22] R. L. Varner et al., Phys. Rep. 201, 57 (1991).

[23] R. C. Johnson and P. J. R. Soper, Phys. Rev. C 1, 976 (1970).

[24] J. A. Tostevin, University of Surrey version of the code TWOFNR (of M. Toyama, M. Igarashi, and N. Kishida) and code FRONT (private communication).

[25] K. Minomo, M. Kohno, K. Yoshida, and K. Ogata, Phys. Rev. C 96, 024609 (2017). 